\title{
Child Abuse and Addiction in Obesity
}

\author{
Michael AB Naafs* \\ Naafs International Health Consultancy, Nederland \\ *Corresponding author: Michael ABN, Naafs International Health Consultancy, Nederland, Europe
}

Submission: 悳 November 14, 2017; Published: 眥 May 31, 2018

\begin{abstract}
In this mini-review the contribution of adverse child effects (ACEs) to obesity and food addiction (FA) is discussed. The concept of FA, its underlying mechanisms and the varying prevalences of FA are reviewd. Its relationship to eating disorders as binge eating, bulimia and substance use disorder is seen from the viewpoint of emotional abuse(EA), emotional neglect(EN) and intimate partner violence (IPV) in childhood. Finally, the therapeutic implications of FA and eating disorders are considered, with an emphasis of various forms of cognitive behavior therapy (CBT) and evolving mHealth CBT.

Abbreviations: ACEs: Adverse Child Effects; FA: Food Addiction; EA: Emotional Abuse; EN: Emotional Neglect; IPV: Intimate Partner Violence; CBT: Cognitive Behavior Therapy; ACEs: Adverse Childhood Experiences; PTSD: Post Traumatic Stress Disorder; YFAS: Yale Food Addiction Scale; BMI: Body Mass Index; PR: Prevalence Ratio; SUD: Substance Use Disorder; BED: Binge Eating Disorder; BN: Bulimia Nervosa; IPV : Intimate Partner Violence; CBT: Cognitive Behavioral Therapy; BWLT: Behaviour weight loss treatment; BWL: Behavioral Weight Loss; ACT: Acceptance and Commitment Therapy
\end{abstract}

\section{Introduction}

Child abuse is associated with being obese as an adult. There is a clear positive dose-response relationship between all 4 types of abuse, including physical, emotional, sexual and general abuse. Hemmingsson et al. [1] concluded from an extensive review of 23 cohort studies ( 4 prospective, 19 retrospective.), with $n=112.708$ participants, that adverse childhood experiences (ACEs) play a major role in obesity development, potentially by inducing mental and emotional pertuberations, maladaptive coping responses, stress, inflammation and metabolic disturbances.

The CDC-Kaiser Permanente Adverse Childhood Experiences (ACE) Study is one of the largest, on-going, investigations in childhood abuse and neglect and later-life health and well-being. The original study started in 1995 at the Kaiser Permanente in San Diego, California, by Fellitti et al. [2] who examined the ACE catogories of childhood physical, sexual, and emotional abuse, childhood physical and emotional neglect, witnessing domestic violence as a child and living with a substance abusing, mentally ill, or incarcerated household member as a child. In this study 60 plus percent of respondents $(n=17.000)$ had at least one ACE,12,6 percent had four or more. Things were going wrong from 4 or more ACEs. As the number of ACEs increases the risk for outcomes, such as heart disease, depression, cancer, smoking, substance use, decreased parenting capability, and obesity increases. The calculated risk of obesity is $34 \%$ higher among adults, who were abused as children, than among adults, who were not. Split into categories, physical abuse during childhood increased the risk of obesity in adulthood by $28 \%$ emotional abuse by $36 \%$, sexual abuse by $31 \%$ and general abuse by $45 \%$ [2]. Probably such stressful experiences increase the risk through emotional factors, which can trigger maladaptive coping responses. The mechanisms involved are increased stress, negative mental and emotional patterns, and poor mental health. These factors impact negatively on appetite regulation, metabolism, eating behavior, sleep, inflammation and cognitive function, which in turn pave the way for obesity [3]. Not everyone, who is subjected to abuse will be obese, and not all obese individuals have been abused. The cause of obesity is multifactorial, such as physical, psychological and social health problems and metabolic, neurohumoral and genetic factors [4]. Anyway obesity is more than "overfeeding and lack of control".

However, some similarities with alcohol and substance abuse were observed. Animal studies suggest, that stress-related consumption of palatable food can become addiction-like in nature [5-7]. A cross sectional study by Hirth et al. [8] found PTSD ( Post Traumatic Stress Disorder) to be associated with increased consumption of fast food and soda in young women. These findings are suggestive of the use of food as self-medication. They are consistent with animal models, indicating that stress may provoke increased consumption of high-fat/high-sugar food, leading to weight gain [9-12]. These studies, animal and clinical have led to public health interest in the potential role of food addiction in the obesity epidemic [13]. Childhood abuse is strongly associated with adult reports of food addiction [14]. In this mini-review the concept 
of food addiction, the underlying mechanisms, its relation with child abuse and substance abuse and the therapeutic implications, will be discussed.

\section{Food Addiction}

The term addiction is applied to excessive ingestion of substances, leading to physical dependence, characterized by tolerance and withdrawal symptoms. Compulsive engagement in behaviors, such as gambling, sex and eating, was not considered a true addiction, because the drive to engage in these behaviors, was considered purely psychological. The conceptual model of substance addictions has begun to change, with an increasing emphasis of the behavior of substance use, rather than the chemical properties themselves [15]. Despite the proposed associationof addictive-like eating with obesiy, it is highly unlikely, that all obese individuals are addicted to food. Also a normal body weight does not equates to a healthy relation with food [16]. To operationalize the addiction-like eating behavior, the Yale Food Addiction Scale (YFAS), translates the substance-diagnostic criteria in a psychometric tool to apply to the consumption of highly palatable foods $[17,18]$. Elevated scores on the YFAS have been linked to more frequent binge-eating episodes, [19-21]. Elevated impulsivity [20], increased depression, [22], higher rates of craving [23], reduced weight loss in response to treatment [17], and an elevated weight regain after bariatric surgery [24]. In addition, addictive eating in both lean and obese people is related to neural activation patterns, observed in other addictive disorders [25].

The prevalence of food addiction (FA) was examined in 2 cohorts of the Nurse Health-2 study ( $n=134,175$ women). Overall $5,8 \%$ of the women surveyed, met the criteria for food addiction by YFAS. The prevalence of FA was $8,4 \%$ in the younger cohort of women, aged 45-64 years, and 2,7\% inthe older cohort of women aged 62-88 years. Body mass index (BMI) greater than 35 (normal $18,5-22,9$ ) was associated with FA with a prevalence ratio (PR) of $15,83(95 \% \mathrm{CI} ; 12,58,19,91)$ in the younger cohort of women and a PR of 18,41 (95\% CI;11,63-29,14) in the older cohort of women. FA prevalence was 2 fold higher in women, who reported depression [16]. Prevalence of FA was $25 \%$ in a study of Davis et al. [26] of obese partcipants. Those who received a food addiction diagnosis, did not differ from non-food addicted participants. The prevalence of FA is increased in obese individuals, and even more so in patients with binge-eating disorder. However the prevalence of FA is not sufficient to account for the obesity epidemic. Conversely, a high prevalence of FA can also be found in under-normal and overweight individuals [27]. Hardy et al. [28] found in a cross-sectional study of patients at risk for PTSD, that women with a substance use disorder (SUD) or FA have similar PTSD and depression symptoms profiles and exhibited more problems with emotional dysregulation, as compared to women with no addictions.

The validity of the clinical assessment of FA as a clinical research tool has not been established yet, despite numerous reports. The assessment of FA largely depends on self-identification, using BMI as a proxy, or administered non-validated questionnaire tools. This leads to variations in reports of FA prevalence. In a meta-analysis of 25 eligible studies ( $n=196.211$, predominantly female), the weighted mean prevalence of YFAS food addiction diagnosis was $19,9 \%$. FA was found to be higher in adults aged $>35$ years, females and overweight participants [29]. There are few studies that evaluate the neural correlates of food in the subgroup of FA obese and the non-FA obese Non-invasive long-term neuromodulation studies have not been performed. Thus even if results look promising, they still have to be considered prelimanary [30].

\section{Binge-Eating}

Patients suffering from Binge Eating Disorder (BED) shows an eating pattern, that is characterized by recurrent episodes, during which they ingest large amounts of food in a discrete period of time. These patients experience a subjective loss of control over their eating behavior. As BED patients do not regurlarly compensate for caloric intake, many patients are overweight and obese [31,32]. It is believed, that BED might represent a phenotype, within the obesity spectrum, that is characterized by increased impulsivity [33-35].

Giel et al. [36] showed in a review of the literature, that rashspontaneous behavior ("no matter the consequences") to food is increased in BED, while food-specific reward sensitivity is also increased in obese individuals without BED. However, food addiction (FA) has a high overlap with BED and obesity [37] and is positively related to increased reward sensitivity and inhibitory control [38-42]. Further research should disentangle the concepts and mechanisms of BED, obesity and food addiction.

To date research has focused on the role of child physical and sexual abuse in eating-related pathology. Less attention has been given tot he role of emotional abuse (EA), emotional neglect (EN) and intimate partner violence (IPV) [43]. Caslimi et al. [44] found a significant and positive association between childhood EA and bulimia nervosa (BN) and BED, calling into question the disproportionate focus on physical and sexual abuse as risk factors. With respect to anorexia nervosa these authors found no signifcant association between anorexia nervosa and childhood exposure to EA [44] EN may have a specific relationship to different forms of eating-disorder pathology, whch are distinct from the impacts of EA. Emotional neglect may be more strongly associated with bingeing behaviors and EA more strongly associated with bingepurge cycles [45].

Generally, a large proportion of adults with eating disorders report EA, EN, or child exposure to IPV, but there is a paucity of high-quality evidence about these relationships. The prevalence of IPV in relation to childhood exposure is not known. Prevalences of EA and EN range from $21 \%$ to $66 \%$ respectively. Samples included predominantly white women and the methodological quality of the available literature is generally low [43].

\section{Obesity and Comorbid Drug Abuse}

Risk behaviors as poor diet, physical inactivity, alcohol and tobacco use are believed to be associated with higher body mass 
index (BMI), trajecting across lifespan [46]. Binge drinking and alcohol, tobacco and marijuana use were positively associated with BMI [47]. Despite the high prevalence of illicit drug use (eg, narcotics, amphetamines, opiates and other prescription drugs) in the U.S. data about the associations between these drugs and BMI are scant. Blackstone et al. [48] examined data from the 20092010 Health Behavior in School-Aged Children study $(n=10.295$ students; Grades 6-10). There were 51,4\% male and 48,6\% female participants; $18,3 \%$ were at risk of being overweight, according to adjusted BMI and 13,8\% were overweight. The percentage of students consuming alcohol in the past 30 days was $25,1 \%$. Illicit drug use in the past 12 months was $14,8 \%$ of the 1907 10th graders asked, and the percentages of students, who had smoked tobacco or marijuana at least once in their lifetime were 15,9\% and 14,3\% respectively. The positive relationship between the use of illicit drug use and BMI proved to be largely due to smoking. When adjusted for smoking this relationship was not significant, anymore.

The research on the relationship between obesity and substance use is most remarkable for its inconsistency. Both positive, negative and no associations have been reported in the literature. Meule, stated that in most studies the specific type of the substance misuse is not defined. Instead of focusing on BMI, it may be more promising to examine the specific kind of eating behavior in relation to substance use. Also, it may be more appropriate to direct research on the relationship between eating disorders and eating topography, than on coprevalence data [49].

A recent PhD thesis by Courtney CL [50] studied a high-risk forensic in-patient population $(n=190)$ and found no associations between ACEs scores and health risk behaviors. The lack of significance in these relationships, suggests that ACEs are less singularly predictors of chronic illness, within this population.

\section{Therapeutic Implications}

Most weight loss research focuses on weight, as the primary outcome and less of the effects of weight loss interventions on physiological, psychological and eating disorders, from these interventions. Peckmezian et al. [51] reviewed 134 studies, that met all these criteria. Lifestyle interventions had the strongest evidence base as a first line approach, with escalation to pharmacotherapy and bariatric surgery in more severe or complicated cases. Quality of life was the most common psychological outcome measure and improved in all cases, where it was assessed, across all intervention types. Behavioral, psychological and lifestyle interventions for weight loss led to improvements in cognitive restraint, control over eating and binge eating, while bariatric surgery led to improvements in eating behavior and body image, that were not sustained over the long term.

Untreated ACEs are supposed to make the treatment of obesity and addictions difficult [2]. Until now, there are no prospective, randomized, controlled trials to support this hypothesis. However, most evidence-based obesity treatment programs integrate different interventions; dietetic, nutritional, physical, behavioral, pharmacological and surgical ones. Such treatment programs are implemented mostly with a team of; endocrinologists, nutritionists, dietitians, physiotherapists, psychologists, psychiatrists and surgeons. Cognitive behavioral therapy (CBT) is traditionally recognized as the best treatment for BED and the most preferred intervention for obesity and could be considered as the first-line treatment among psychological treatments [52]. However, it does not result necessarily in a long term, prolonged and sustained weight loss [53]. Anyway, CBT has the possibility to bring the ACEs at the surface $[54,55]$. Traditional medication protocols and psychological treatments have short term efficacy in comparison with placebo [56,57].

Is CBT clinically effective only in obesity with BED ? [52]. Epidemiological studies showed BED being the most common eating disorder [58,59]. There is a strong association between BED and obesity, even if obesity is not a criterion of BED. Furthermore, there are important differences, between obese binge eaters and obese non-binge eaters $[60,61]$. To complicate matters further emotional neglect (EN) is strongly associated with bingeing behaviors and emotional abuse (EA) with binge-purging cycles, as depicted above [45]. CBT can reduce binge eating episodes significantly, promote days without bingeing, manage eating anf reduce shape and weight concerns, without directly affecting body weight [62]. Follow-up studies to monitor the long-term results are requested, but not available [61-65].

CBT has strongly developed in the management of obesity and eating disorders. Various modifications are offered now. These include "enhanced" treatment (CBT-E), directing exclusively at the psychopathology of eating disorders and a more complex form (CBT-Eb), that also addresses problems as mood intolerance, clinical perfectionism, low self-esteem and interpersonal difficulties [66]. Behavior weight loss treatment (BWLT) or behavioral weight loss (BWL) consider diet, exercise and behavior therapy. Behavior modification strategies include self-monitoring, goal-setting, shaping, reinforcement and stimulus. Their additional value over conventional CBT is not proven yet and data about cost-effectiveness are lacking [67-69]. CBT is also combined with the acceptance and commitment therapy (ACT), which nay have a mindfulness component, too [70-72]. Mindfulness enhancements to a diet-exercise program did not show substantial weight loss in a study by Daubenmier et al. ( $n=194)$ [73]. CBT treatments for obesity are also delivered by mHealth applications in remote settings. These applications have recieved positive results [74-76]. But even if evidence is growing in mHealth for obesity, organizational, technological, economical and philosophical barriers are still present [77]. Unfortunately all these studies have in common again, that they are primarily directed on BMI, and not on the relationship between ACEs and eating behavior or eating topography. As Adrian Meule [23] says "it is still a true can of worms" [49]. An extensive discussion about the role of pharmacology and bariatric surgery in obesity treatments, is beyond the scope of this mini-review. 


\section{Conclusion}

Child abuse shows a clear positive dose-response relationship with he number of adverse child events (ACEs). The calculated risk of obesity is $34 \%$ higher amomg adults, who were abused as a child. While there is a disproportionate attention for physical and sexual abuse, factors as emotional abuse(EA), emotional neglect (EN) and intimate partner violence (IPV) have a more deleterious effect on obesity and eating disorders. EA is significantly associated with bulimia nervosa (BN) and binge eating disorder (BED). EN is more strongly associated with bingeing behaviors and EA more strongly with binge-purge cycles. The positive relationship between the illicit use of drugs and body mass index (BMI) proved to be largely due to smoking. The research on the relationship between obesity and substance use is most remarkable for its inconsistency. Both positive, negative and no associations have been reported in the literature. Most weight loss research focuses on weight as primary outcome and less on the effects on physiological, psychological and eating disorders from interventions. Lifestyle interventions have the strongest evidence base as a first line approach, with escalation to pharmacotherapy and bariatric surgery in severe cases. Quality of life improved in all cases, across all intervention types. Untreated ACEs are supposed to make the treatment of obesity and addictions difficult. There are still no prospective, controlled, randomized trials available, that support this hypothesis. Combined with lifestyle interventions, exercise, CBT has a positive short term effect compared to placebo. Reliable long-term results of CBT ( 3 years or more) are lacking. CBT has developed various forms in the treatment of obesity, that have to prove their value over conventional CBT, yet. This is also the case for mHealth CBT. Generally, the research in obesity has many flaws and is methodologically of low quality, relying on predominantly white women, self identification,using BMI as a proxy for food addiction.

\section{References}

1. Hemmingsson E, Johansson K, Reynisdotir S (2014) Effects of childhood abuse on adult obesity: a systematic review and meta-analysis. Obes Rev 15(11): 882-893.

2. Felliti VJ, Anda RF, Nordenberg P, Williamson DF, Spitz AM, et al (1998) Relationship of childhood abuse and household dysfunction to many of the leading causes of death in adults: The Adverse Childhood Experiences (ACE) Study. Am J Prev Med 14(4): 245-258.

3. Hemmingsson E (2014) A new model of the role of psychological and emotional distress in promoting obesity: conceptual review with implications for treatment and prevention. Obes Rev 15(9): 769-779.

4. Karnik S, Kanekar A (2012) Childhood Obesity: A global publc health crisis. Int J Prev Med 3(1): 1-7.

5. Adam IC, Epel ES (2007) Stress, eating and the reward system. Physiol Behav 9(14): 449-458.

6. Goeders NE (2002) Stress and cocaine addiction. J.Pharmacol ExpTher 301(3): 785-789.

7. Johnson PM, Kenny PJ (2010) Dopamine D2 receptors in addiction-like reward dysfunction and compulsive eating in obese rats. Nat Neurosci 13(5): 635-641.
8. Hirth JM, Rahman M, Berenson AB (2011) The association of posttraumatic stress disorder with fast food and soda consumption and unhealthy weight loss behaviors among young women. J Womens Health (Larchmt) 20(8): 1141-1149.

9. Brewerton TD (2011) Posttraumatic stress disorder and disordered eating: food addiction as self-medication. J Womens Health 20(8): 11331134.

10. Corwin RL, Avena NM, Boggiano MM (2011) Feeding and reward: perspectives from three rat models of binge eating. Physiol Behav 104(1): 87-97.

11. Rowland NE, Antelman SM (1976) Stress-induced hyperphagia and obesity in rats: a possible model for understanding human obesity. Science 191(4224): 310-312.

12. Dallmann MF, Pecoraro N, Akana SF, La Fleur SE, Gomez F, et al. (2003) Chronic stress and obesity: a new view of "comfort food". Proc Natl Acad Sci USA 100(20): 11696-11701.

13. Gearhardt AN, Grilo CM, Di Leone PJ, Brownell KD, Marc Potenza N (2011) Can food be addictive? public health and policy implications. Addiction 106 (7): 1208-1212.

14. Mason SM, Flint AJ, Field AE, Austin SB, Rich EJW (2013) Abuse victimization in childhood or adolescence and risk of food addiction in adult women. Obesity 21(12): 775-781.

15. Barry D, Clarke M, Petry NM (2009) Obesity and its relationship to addictions:Is overeating a form of addictive behavior ? Am J Addict 18(6): 439-451.

16. Flint AJ, Gearhardt AN, Corbin WR, Brownell KD, Field AE (2014) Foodaddiction scale measurement in 2 cohorts of middle-aged and older woman. Am J Clin Nutr 99(33): 578-586.

17. Gearhardt AN, Corbin WR, Brownell KD (2009) Preliminary validation of the Yale Food Addiction Scale. Appetite 52(2): 430-436.

18. Gearhardt AN, Corbin WR, Brownell KD (2009) Food Addiction: an examination of the diagnostic criteria for dependence. J Addict Med 3(1): 1-7.

19. Burmeister JM, Himman N, Koball A, Hoffmann DA, Carels RA (2013) Food addiction in adults seeking weight loss treatment: Implications for psychosocial health and weight loss. Appetite 60(1): 103-110.

20. Davis C, Curtis C, Levitan RD, Carter JC, Kaplan AS (2011) Evidence that "food addiction"is a valid phenotype of obesity. Appetite 57(3): 711-717.

21. Gearhardt AN, White MA, Masheb RM, Carlos MG (2013) An examination of food addiction in a racially diverse sample of obese patients with binge eating disorder in primary care settings. Compr Psychiatry 54(5): 500-505.

22. Eichen DM, Lent MR, Goldbacher E, Foster GD, et al. (2013) Exploration of "Food Addiction" in overweight and obese treatment-seeking adults. Appetite 67: 22-24.

23. Meule A, Kubler A (2012) Food cravings in food addiction: the distinct role of positive reinforcement. Eat Behav 13(3): 252-255.

24. Clark SM, Saules KK (2013) Validation of the yale food addiction scale among a weight-loss surgery population. Eat Behav 14(2): 216-219.

25. Brooks SJ, Cedermaes J, Schioth HB (2013) Increased prefrontal and hippocampal activation with reduced dorsolateral ,prefrontal and insular cortex activation to food images in obesity: a meta-analysis of fMRI studies. PLoS One 8(4): 8e60393.

26. Davis C, Carter JC (2009) Compulsive overeating as an addiction disorder: A review of theory and evidence. Appetite 53(1): 1-8. 
27. Meule A (2011) How prevalent is "food addiction" ? Front Psychiatry 2 61.

28. Hardy R, Fani N, Jovanovic T, Michopoulos V, et al. (2018) Food addiction and substance addiction in women: Common clinical characteristics. Appetite 120: 367-373.

29. Pursey KM, Stanwell P, Gearhardt AN, Collins CE, Burrows TL (2014) The prevalence of food addiction as assessed by the yale food addiction scale: A systematic review. Nutrients 6(10): 4552-4590.

30. De Ridder D, Manning P, Cape G, Sven V, Berthold L, et al. (2016) Pathophysiology-Based Neuromodulation Neuropathology of Drug Addictions and Substance Misuse. Academic Press, pp. 14-24

31. American Psychiatric Association. (2013) Diagnostic and Statistical Manual of Mental Disorders: DSM-5. American Psychiatric Association Washington, DC, USA

32. Kessler RC, Berglund PA, Chiu WT, Deitz AC, Hudson JI, et al. (2013) The prevalence and correlates of binge eating disorder in the WHO world mental health surveys. Biol Psychiatry 73(9): 904-914.

33. Gerlach G, Herpertz S, Loeber S (2015) Personality traits and obesity: A systematic review. Obes Rev 16(1): 32-63.

34. Kessler RM, Hutson P, Herman BK, Potenza MN (2016) The neurobiological basis of binge eating disorder. Neurosci Biobehav Rev 63: 223-238.

35. Schag K, Schönleber J, Teufel M (2013) Food-related impulsivity in obesity and binge eating disorder: A systemic review. Plos One 14(6): 477-95.

36. Giel KE, Teufel M, Junne F, Zipfel S, Schag K (2017) Food-related impulsivity in obesity and binge eating disorder:A systematic update of the evidence. Nutrients 9(11): E1170.

37. Gearhardt AN, White MA, Potenza MN (2011) Bnge eating disorder and food addiction. Curr Drug Abuse Rev 4(3): 201-207.

38. Gearhardt AN, Yokum S, Orr PT, Stice E, Corbin WR (2011) Neural correlates of food addiction. Arch Gen Psychiatry 68(8): 808-816.

39. Hsu JS, Wang PW, Ko CH, Hsieh TJ, Chen CY, et al. (2017) Altered brain correlates of response inhibition and error processing in females with obesity and sweet food addiction: A functional magnetic imaging study. Obes Res Clin Pract 11(6): 677-686.

40. Imperatori C, Fabbricatore M, Innamorati M, Farina B, Quintiliani MI, et al. (2015) Modification of EEG functional connectivity and EEG power spectra in overweight and obese patients with food addiction: An eLORETA study. Brain Imaging Behav 9(4): 703-716.

41. Loxton NJ, Tipman RJ (2017) Reward sensitivity and food addiction in women. Appetite 115: 28-35.

42. Vander Broek Stice L, Stojek MK, Beach SR, vanDellen MR, MacKillop J (2017) Multidemensional assessment of impulsivity in relation to obesity and food addiction. Appetite 112: 59-68.

43. Kimber M, McTavish JR, Boven A, Alison Boven, Sana Gill, et al. (2017) Consequences of child emotional abuse, emotional neglect and exposure to intimate partner violence for eating disorders: a systematic critical review. BMC Psychol 5: 33.

44. Caslimi M, Bartoli F, Crocamo C, Dakanalis A, Clerici M, et al. (2016) Disentangling the association between child abuse and eating disorders: a systematic review and meta-analysis. Psychosom Med 78(1): 79-90.

45. Moulton SJ, Newman E, Power K, Swanson V, Day K (2015) Childhood trauma and eating psychopathology: a mediatory role for dissociation and emotion dysregulation? Child Abuse Negl 39: 167-174.

46. Huang DYC, Lanza HI, Wright Volel K, Anglin MD (2013) Developmenta trajectories of childhood obesity and risk behaviors in adolescence. J
Adolesc 36(1): 139-148.

47. Pasch KE, Nelson MC, Lytle LA, Moe SG, Perry CL (2008) Adoption of risk-related factors through early adolescence: Associations with weight states and implications for causal mechanisms. J.Adolesc. Health 43(4): 387-393.

48. Blackstone SR, Hermann LK (2015) Relationships between illicit drug use and body mass index among adolescents. Health Educ Behav 43(1): $21-24$

49. Meule A (2014) the relation between body mass index and substance use: a true can of worms. Innov Clin Neurosci 11(3-4): 11-12.

50. Cook CL (2017) Early childhood adversity and chronic illness: an examination of a high risk-forensic-inpatient population. Electronic Thesis and Dissertations, pp. 1-105.

51. Peckmezian T, Hay P (2017) A systematic review and narrative synthesis of interventions for uncomplicated obesity: weight loss,well-being,and impact on eating disorders. J Eat Disord 5: 1-15.

52. Moffitt R, Haynes A, Mohr P (2015) Treatment beliefs and preferences for psychological treatments for weight management. J Clin Psychol 71(6): 584-596.

53. Grilo CM, Masheb RM, Wilson GT, Gueorguieva R, White MA (2011) Cognitive behavioral therapy, behavioral weight loss and sequential treatment for obese patients with binge-eating disorder: a randomized controlled trial. J Consult Clin Psychol 79(5): 675-685.

54. Castelnuevo G, Pietrabissa G, Manzoni GM, Cattivelli R, Rossi A, et al. (2017) Cognitive behavioral therapy to aid weight loss in obese patients;current perspectives. Psychol Res Behav Manag 10: 165-173.

55. Cohen JA, Mannarino AP (2015) Trauma-focused cognitive behavioral therapy for traumatize reasd children and families. Child Adolesc Psychiatr Clin N Am 24(3): 557-570.

56. Reas DL, Grilo CM (2008) Review and meta-analysis of pharmacotherapy for binge-eating disorder. Obesity 16(9): 2024-2038.

57. Wilson GT, Grilo CM, Vitousek KM (2007) Psychological treatment of eating disorders. Am Psychol 62(3): 199-216.

58. Hudson JL, Hirip E, Pope HG, Kessler RC (2007) The prevalence and correlates of eating disorders in the National Comorbidity Survey Replication. Biol Psychiatry 61(3): 348-358.

59. Jacobi C, Hayward C, de Zwaan M, Kraemer HC, Agras WS, et al. (2004) Coming to terms with risk factors for eating disorders: applications of risk terminology and suggestions for a general taxonomy. Psychol Bull 130(1): 19-65.

60. Bulik CM, Reichborn Kjennerud T (2003) Medical morbidity in binge eating disorder. Int J Eat Disord 34(Suppl): S39-S46.

61. Vanderlinden J, Adriaensen A, Vancampfort D, Pieters G, Probst M (2012) A cognitive behavioral therapeutic program for patients with obesity and binge eating disorder:short- and long-term follow-up data of a prospective study. Behav Modif 36(5): 670-686.

62. Vocks S, Tuschen Caffier B, Pietrowsky R, Rustenbach SJ, Kersting A et al. (2010) Meta-analysis of the effectiveness of psychological and pharmacological treatments for binge eating disorder. Int J Eat Disord 43(3): 205-217.

63. Treasure J, Claudino AM, Zucker N (2010) Eating disorders. Lancet 375(9714): 583-593.

64. Brownley KA, Berkman ND, Sedway JA, Lohr KN, Bulik CM (2007) Binge eating disorder treatment: a systematic review of randomized controlled trials. Int J Eat Disord 40(4): 337-348.

65. Dingemans AE, Bruna MJ, van Furth EF (2002) Binge eating disorder: review. Int J Obes Relat Metab Disord 26(3): 299-307. 
66. Fairburn CG, Cooper Z, Doll HA, O'Connor ME, Bohn K, et al. (2009) Transdiagnostic cognitive behavior therapy for patients with eating disorders; a two-site trial with 60- week follow-up. Am J Psychiatry 166(3): 311-319.

67. Munsch S, Biedert E, Meyer A, Michael T, Schlup B, et al. (2007) A randomized comparison of cognitive behavioral therapy and behavioral weight loss treatment for overweight individuals with binge eating disorder. Int J Eat Disord 40(2): 102-113.

68. Khaylis A, Yiaslas T, Bergstrom J, Gore Felton C (2010) A review of efficacious technology-based weight loss interventions: five key components. Telemed J E Health 16(9): 931-938.

69. Palavros MA, Hay P, Touyz S, Amanda Sainsbury, Felipe da Luz, et al. (2015) Comparing cognitive behavioral therapy for eating disorders integrated with behavioral weight loss therapy to cognitive behavioral therapy-enhanced alone in overweight or obese people with bulimia nervosa or binge eating disorder: study protocol for a randomized controlled trial. Trials 16: 1-578.

70. Cattivelli R, Pitrabissa G, Ceccarini M, Chiara AMS, Valentina Villa, et al. (2015) ACTonFOOD: opportunities of ACT to address food addiction. Front Psychol 6: 396.

71. Lilis J, Niemeier HM, Thomas JG, Unick J, Ross KM, et al. (2016) A randomized trial of an acceptance-based behavioral intervention for weight loss in people with high internal disinhibition. Obesity 24(12): $2509-2514$
72. Forman EM, Butryn ML, Manasse SM, Crosby RD, Goldstein SP, et al. (2016) Acceptance-based versus standard behavioral treatment for obesity: results from the mind your health randomized,controlled trial. Obesity 24(10): 2050-2056.

73. Daubenmier J, Moran PJ, Kristeller J, Acree M, Bacchetti P, et al. (2016) Effects of a mindfulness-based weight loss intervention in adults with obesity: A randomized clinical trial. Obesity 24(4): 794-804.

74. Turner McGrievy GM, Beets MW, Kaczynski AT, Barr Anderson DJ, Tate DF (2013) Comparison of traditional versus mobile app-selfmonitoring of physical activity and dietary intake among overweight adults participating in a mHealth weight loss program. J Am Med Inform Assoc 20(3): 513-518.

75. Burke LE, Styn MA, Sereika SM, Conroy MB, Ye L, et al. (2012) Using mHealth technology to enhance self-monitoring for weight loss: a randomized trial. Am J Prev Med 43(1): 20-26.

76. Sockalingom S, Cassin SE, Wnuk S, Du C, Jackson T, et al. (2017) A pilot study on telephone cognitive behavioral therapy for patients six months post-bariatric surgery. Obes Surg 27(3): 670-675.

77. Mohammadzadeh N, Sadfari R (2014) Patient monitoring in mobile health: opportunities and challenges. Med Arch 68(1): 57-60.
Creative Commons Attribution 4.0 International License

For possible submissions Click Here

\section{Submit Article}

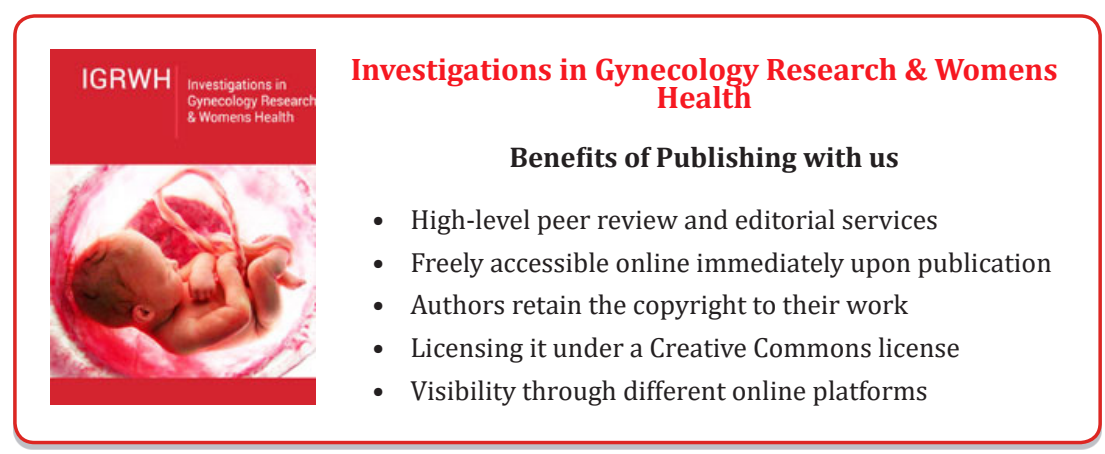

